\title{
Planeación estratégica de marketing y su importancia en las empresas
}

\section{Strategic marketing planning and its importance in companies}

Silvia Alexandra Jaramillo Luzuriaga

Lorena del Cisne Tacuri Peña

Daniela Alexandra Trelles Ordóñez

Universidad Internacional del Ecuador, Ecuador

Autor para correspondencia: sijaramillolu@uide.edu.ec, lotacuripe@uide.edu.ec, datrellesor@uide.edu.ec

Fecha de recepción: 23 de agosto de 2018 - Fecha de aceptación: 01 octubre de 2018

Resumen: El presente artículo tiene como objetivo analizar la planeación estratégica de marketing y su importancia en las empresas, cuyo alcance es mejorar los niveles competitivos y por ende generar mayores utilidades a través de la aplicación adecuada de la planeación, estrategias de marketing, objetivos, conceptos y procedimientos, los mismos que facilitan a los gerentes a la toma de decisiones futuras y llevar una adecuada administración. En esta investigación se utilizó la metodología que incluye información primaria y secundaria, estableciendo como métodos principales: el inductivo, deductivo y estadístico; así como las técnicas para la obtención de la información como es la observación directa a las empresas y las encuestas que se aplicó a una muestra de 260 empresas privadas de la ciudad de Loja. En los resultados obtenidos se analizó como se desenvuelven las empresas con respecto a la planeación estratégica de marketing, por lo que se logró determinar que algunas empresas si aplican algún tipo de planeación para el desarrollo de sus actividades, sin embargo no la aplican adecuadamente, en unos casos se debe a que son negocios familiares y lo hacen empíricamente, lo que no les permite tener un buen desenvolvimiento empresarial. Por tanto, se concluyó que en las empresas la planeación estratégica es la base fundamental, ya que gracias a ella se puede prever alguna situación adversa e implementar planes de acción en caso de contingencias, además de que al planear un horizonte empresarial se puede lograr un objetivo con más calma ya que al planear se revelan los posibles errores y las ventajas con las que se cuenta al momento de realizar un proyecto, es decir, la planeación estratégica conlleva al logro de los objetivos y metas planteadas, según la amplitud y magnitud de las empresas.

Palabras Claves: planeación; estrategia; marketing; objetivos; empresas

Abstract: This article has as objective to analyze the strategic planning of marketing and its importance in the companies, whose scope is to improve the competitive levels and therefore generate higher profits through the appropriate application of the planning, strategies of marketing objectives, concepts and procedures, which make it easier for managers to make future decisions and take a proper administration. This research was used in the methodology which includes primary and secondary information setting as main methods: the inductive, deductive and statistical; as well as the techniques for obtaining the information as it is direct observation to companies and surveys that is applied to a sample of 260 companies from the city of Loja. The 
results obtained were analyzed as the strategic planning of marketing companies, they operate by what was achieved to determine that some companies if they apply some kind of planning for the development of its activities, however not the applied properly, in a few cases is that they are family businesses and do so empirically, not allowing them have a good business development. Therefore, it was concluded that in enterprises strategic planning is the fundamental basis, because thanks to it you can anticipate any adverse situation and implement plans of action in the event of contingencies, in addition to planning a business horizon can achieve a goal more calmly since planning reveal errors and the advantages it has at the time of a project, i.e., strategic planning leads to the achievement of the objectives and targets raised, according to the extent and magnitude of the companies.

Key Words: planning; strategy; marketing; goals; business

\section{Introducción}

Este artículo tiene como propósito dar a conocer la importancia de la planeación estratégica de marketing en las empresas, actualmente las empresas se enfrentan a un mercado de grandes exigencias tales como la calidad, garantía, variedad, de crédito, ubicación, la influencia de aspectos externos como cambios principales de políticas de gobierno y economía mundial, siendo ante ello imprescindible el aplicar una adecuada planificación estratégica de marketing para un buen desenvolvimiento empresarial.

La importancia de la planeación estratégica de marketing radica principalmente en que ayuda a los directivos de las empresas a la toma de decisiones en la detección de errores y los lineamientos a seguir para poder corregirlos, además de establecer los objetivos a ser alcanzados en un tiempo establecido. Las estrategias de marketing proporcionan un marco de referencia para la actividad organizacional que pueda conducir a un mejor funcionamiento y una mayor sensibilidad.

Es así que en las empresas la planificación estratégica de marketing representa el punto de partida para un excelente desempeño dentro del campo en el que este enfocada, ya que une las fortalezas comerciales con las oportunidades de mercado y brinda una dirección para cumplir con los objetivos.

Hoy en día la mayoría de las empresas si tienen conocimientos respecto a la implementación de un plan estratégico de marketing que incluya metas y objetivos para cada área del negocio, entre las que se encuentran la organización, la administración, la mercadotecnia, las ventas, los clientes, los productos, los servicios y las finanzas. Estas áreas comerciales se distribuyen en un sistema integrado que permite que la organización pueda trazar una ruta de manera exitosa y encaminarse a cumplir sus metas.

Las empresas reconocen la necesidad de la planeación estratégica de marketing para su crecimiento y bienestar a largo plazo, ayudando a orientar las energías y recursos hacia las características de alta prioridad. Es decir, propicia el desarrollo de las empresas al establecer métodos de utilización racional de los recursos, reduciendo los niveles de incertidumbre que se pueden presentar en el futuro, y por ende preparando a la empresa para hacer frente a las contingencias que se presenten, además condiciona a la empresa al ambiente que la rodea, y por ultimo reduce los riesgos y aprovecha al máximo las oportunidades. 


\section{Marco teórico}

\section{La planificación}

Para Munch (2011) hablar de planeación significa destacar que este es esencial para un correcto funcionamiento de cualquier grupo social, pues, previenen las contingencias y los cambios logrando establecer medidas necesarias para afrontarlas. Entre algunas se menciona las siguientes: promueve la eficiencia al eliminar la improvisación, reduce los riesgos y aprovecha las oportunidades, da el rumbo y encamina a la empresa aprovechando sus recursos, las decisiones se basan en hechos y no en emociones.

La planificación implica seleccionar misiones y objetivos, así como las acciones necesarias para cumplirlos, y requiere por lo tanto de la toma de decisiones; esto es, de la elección de cursos de acciones futuras a partir de diversas alternativas (Koontz, 2010).

\section{Planificación estratégica}

La planificación estratégica es un proceso de decisión que persigue como objetivo que la empresa esté permanentemente adaptada a su entorno, de la manera más adecuada. Según Kotler (2000) la planificación consiste en decidir hoy lo que va a hacerse en el futuro, es decir, comprende la determinación de un futuro deseado y las etapas necesarias para realizarlo.

Es un proceso que permite asegurar la competitividad presente y futura de quien la impulsa analizando la propia situación interna y anticipando la evolución del entorno, concreta las ideas en planes y programas de actuación, definidos por el tiempo y el espacio, formulados en tiempos definidos, medibles y verificables en términos de costo, inversión y resultados. (Arguin, 2000, pág. 34)

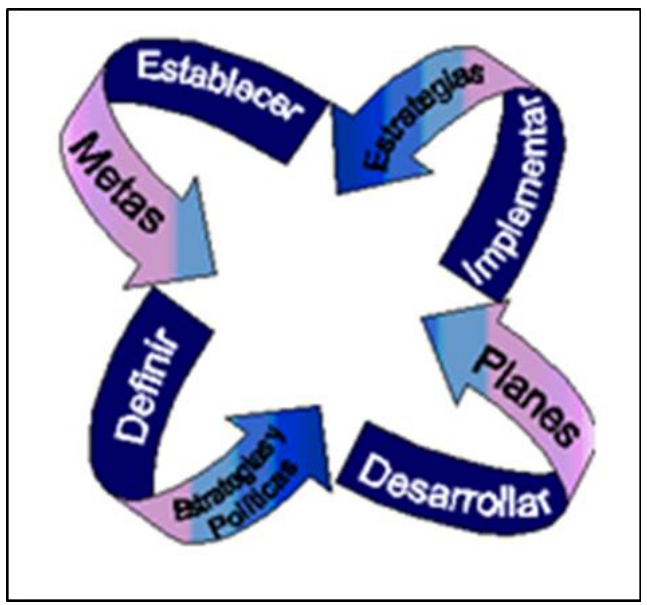

Figura 1. Planificación estratégica Fuente: http://www.eoi.es/blogs/juanadoricelcepeda/files Elaboración: La Autora.

El proceso de planificación estratégica se diferencia porque se marca dentro de un ámbito más amplio que es de la gestión empresarial, entendiéndose como tal al conjunto de decisiones y acciones de los mandos medios y altos de una institución que se ejecutan para el cumplimiento de los objetivos y servicios que se ha propuesto. 


\section{Marketing}

"Es el proceso social y administrativo por el cual los grupos e individuos satisfacen sus necesidades al crear e intercambiar productos, bienes y servicios" (Kotler \& Armstrong, 2004, pág. 43).

\section{Estrategias de marketing mix}

Las estrategias de marketing mix se inician con la planeación, fijación de precios, distribución y promoción de ideas, bienes y servicios orientados a satisfacer los objetivos individuales y de la empresa, así como también satisfacer las necesidades del mercado al cuál se han proyectado los componentes antes mencionados. Se evidencia lo que comúnmente se denomina mezcla de mercadeo o marketing mix. Los empresarios cuentan con una serie de herramientas útiles para ingresar a un mercado competitivo estas son las 4 pes: Producto, Precio, Plaza y Promoción. De esta manera el empresario puede aplicar estas estrategias para satisfacer las necesidades de sus clientes. (Echeverri, 2008, pág. 66)

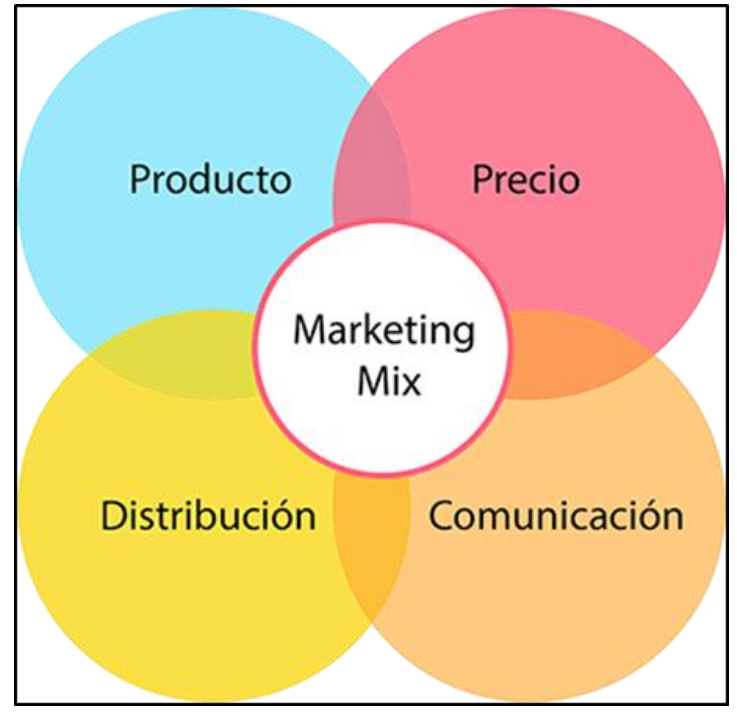

Figura 2. Marketing mix

Fuente: https://www.google.com/imgres?imgurl=

Elaboración: La Autora.

\section{Plan estratégico de marketing}

Un plan es una serie de pasos o procedimientos determinados, que buscan conseguir un objetivo o propósito. Al proceso para diseñar un plan se le conoce como planeación o planificación, y de manera amplia, la planificación consiste en definir claramente los objetivos y las acciones que se deben tomar para llegar a alcanzarlos (Fred, 1997).

"El plan estratégico de marketing se caracteriza por ser un plan a largo plazo, del cual, se parte para definir las metas a corto plazo" (Ferrel \& Hirt, 2004, pág. 211).

\section{Proceso de la planeación estratégica de marketing}

La planeación estratégica de marketing es un documento escrito que incluye una estructura, los cuales son adaptados a las necesidades de cada empresa u organización. El 
proceso de planificación estratégica de marketing se desarrolla de acuerdo con el esquema expuesto en el siguiente gráfico.

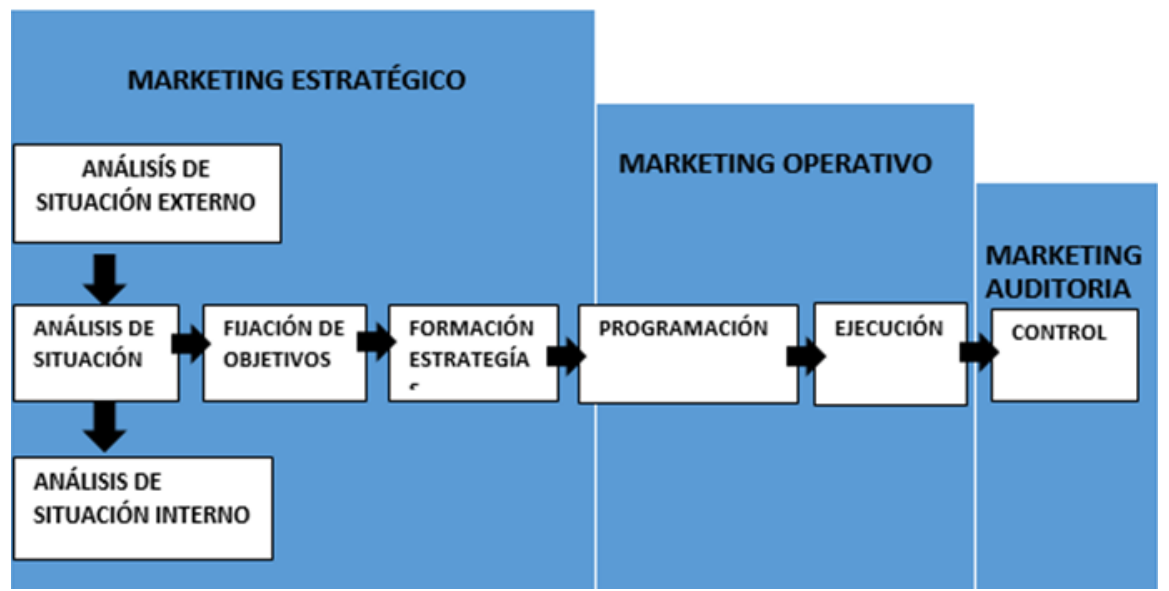

Figura 3. Proceso de la planificación estratégica de marketing

Fuente: "La dirección estratégica de la empresa" Renau Menguzato 2010

Elaboración: La Autora.

Dicho proceso de la planeación estratégica de marketing podría resumirse en cuatro interrogantes clave:

¿Quiénes somos? / Definición de la misión de la empresa.

¿Dónde nos encontramos? / Análisis de la situación.

¿A dónde queremos llegar? / Establecimiento de los objetivos de marketing.

¿Cómo lo conseguiremos? / Formulación de las estrategias de marketing.

La supervivencia de la empresa depende de una adecuada respuesta a estas cuestiones, para lo que hará uso de la planificación estratégica (Renau, 2010).

\section{Establecimiento de los objetivos}

Representan los resultados que la empresa espera obtener, son fines por alcanzar, estableciendo cuantitativamente y determinados para realizarse transcurrido un tiempo específico; para fijar objetivos, la empresa debe identificar sus áreas claves para el éxito o supervivencia. La empresa debe saber en dónde se encuentra y a dónde quiere llegar. El establecimiento de objetivos está basado en los puntos revisados anteriormente, un objetivo debe ser específico, medible, posible y con un horizonte de tiempo para lograrlo. (Cutropía, 2003, pág.6)

\section{Formulación de las estrategias}

La estrategia es el curso de acción general o alternativa que muestra la dirección y empleo general de recursos y esfuerzos para logar objetivos en condiciones ventajosas. "La estrategia es el elemento que determina las metas básicas de la empresa, a largo plazo; así como la adopción de cursos de acción y asignación de recursos para alcanzar las metas" (Rojas, 2004, pág. 112).

Según Porter (2002), para comprender esta etapa en las organizaciones modernas distinguió tres niveles en el proceso de formulación de la estrategia: 
Estrategia corporativa: las organizaciones deben llevar a cabo las cuatro actividades de planificación.

La estrategia de la unidad de negocio: atendiendo a la ventaja competitiva defendible por la empresa y al objetivo estratégico.

La estrategia funcional: una vez que la estrategia corporativa y de unidad de negocio han sido formuladas, se está en condiciones de iniciar la planificación de las distintas áreas funcionales: marketing, producción, finanzas, etc. (Porter, 2002).

\section{Importancia de la planeación estratégica en las empresas}

La planeación estratégica proporciona a los directivos y a los empleados una visión de lo que se puede lograr en un futuro para la empresa en la cual trabajan, así como la tranquilidad y sentido de pertenencia en la organización provocando una motivación y empuje hacia la participación activa, colaboración y trabajo en equipo buscando el logro de los objetivos planteados.

Hoy en día las empresas tienen que estar a la vanguardia de manera constante debido a este mundo ya tan globalizado, y aprovechar al máximo todos los recursos disponibles y alcanzables para su continuidad en el mercado. La tecnología juega un papel muy importante en las organizaciones ya que esta le ayuda e impulsa a ser más eficiente en sus procesos así como en la obtención y/o creación de nuevos productos, por eso es muy importante mantener al personal en constate capacitación.

Thompson, Strickland y Gamble, (2012) en su libro administración estratégica, hace referencia de la importancia que tiene una planeación y como marca la diferencia en las organizaciones:

Lo que separa a una estrategia poderosa de una común es la capacidad de la dirección para forjar una serie de movimientos, tanto en el mercado como en su interior, que aleje a la empresa de sus rivales, incline la balanza a su favor dando razones a los clientes para que prefieran sus productos o servicios, y produzca una ventaja competitiva sustentable sobre sus rivales. (Thompson, Strickland, \& Gamble, 2012, pág. 22)

\section{Materiales y métodos}

\section{Diseño de la investigación}

De acuerdo con el propósito de la investigación, está se enmarcó dentro de un tipo de enfoque cualitativo, explorativo y descriptivo, donde primero se recopiló la información para luego analizar e interpretar los datos recolectados, siendo lo más apropiado para alcance de los objetivos planteados; pues al ser cualitativo es flexible y abierta, se ajusta a sus características, no solo del objeto de estudio, sino también a las condiciones que se circunscribe.

A su vez, tiene un carácter exploratorio, ya que permitió conocer y ampliar el conocimiento sobre el tema a investigar para precisar mejor la planeación estratégica de marketing y su importancia en las empresas, y de esta manera resolver la problemática encontrada en relación a las limitantes que se presentan actualmente en las empresas. 
Dentro del marco mencionado, tiene un carácter descriptivo, ya que permitió describir la situación mediante el estudio e interpretación correcta de la información, a través de la aplicación de las encuestas a las empresas.

\section{Población}

En el proceso investigativo se determinara la población al cual va dirigido el estudio, considerando una primera segmentación demográfica (empresas privadas) y en segunda instancia una segmentación geográfica (parroquias del área urbana de la ciudad de Loja).

Tomando como base los datos registrados en la Cámara de Comercio de Loja hasta mayo 2018, el número de empresas del área urbana de la ciudad de Loja es de 800 empresas privadas.

\section{Tamaño de la muestra}

Luego de haber determinado la población se procedió al cálculo del tamaño de la muestra, donde se tomó como referencia las 800 empresas privadas del área urbana de la ciudad de Loja.

Se calculó la muestra mediante la aplicación de la fórmula matemática, como se describe a continuación:

FÓRMULA:

$\mathrm{n}=$ Tamaño de la Muestra

$\mathrm{Z}=$ Nivel de confianza $(95 \%=1,96$ tabla de distribución anual)

$\mathrm{p}=0,5$ Probabilidad de que el evento ocurra

$\mathrm{q}=0,5$ Probabilidad de que el evento no ocurra

$\mathrm{N}=$ Población (800)

$$
\begin{gathered}
\mathrm{n}=\frac{z^{2} p * q * N}{e^{2}(N-1)+Z^{2} * p * q} \\
\mathrm{n}=\frac{(1,96)^{2} *(0,5) *(0,5) *(800)}{(0,05)^{2}(800-1)+(1,96)^{2} *(0,5) *(0,5)} \\
\mathrm{n}=\frac{768,32}{1,9975+0,9604} \\
\mathrm{n}=\frac{768,32}{2,9579}
\end{gathered}
$$

\section{Entorno}

n=260 Encuestas (empresas del área urbana de la ciudad de Loja)

La investigación se desarrolló en un contexto local como es en las empresas privadas (área urbana) de la ciudad de Loja, cantón Loja, provincia de Loja.

\section{Intervenciones}

En el desarrollo de la presente investigación se utilizó: métodos, técnicas e instrumentos que permitieron el desarrollo adecuado de la misma. Así tenemos:

\section{Métodos}


De entre los métodos de investigación que se utilizó en el presente estudio, tenemos los siguientes:

\section{Método deductivo}

La deducción va de lo general a lo particular. El método deductivo es aquél que parte los datos generales aceptados como valederos, para deducir por medio del razonamiento lógico, varias suposiciones. Este método se lo utilizó en la recopilación de información secundaria para realizar las encuestas y efectuar el análisis e interpretación de resultados.

\section{Método inductivo}

La inducción va de lo particular a lo general. El método inductivo es aquel que a partir de la observación de los hechos particulares se obtiene proposiciones generales, o sea, es aquél que establece un principio general una vez realizado el estudio y análisis de hechos y fenómenos en particular. Este método permitió el análisis de los aspectos más relevantes a través de los procesos analíticos y sintéticos, y obtener las conclusiones principales para la investigación.

\section{Técnicas}

En este tema de investigación se empleó las siguientes técnicas:

\section{La encuesta}

La encuesta es el conjunto de preguntas especialmente diseñadas y pensadas para ser dirigidas a una muestra de población, con el objetivo de conocer la opinión de la gente sobre determinadas cuestiones. Está técnica permitió obtener datos a través de la información proporcionada por los directivos de las empresas sobre cómo se desenvuelven las empresas con respecto a la planeación estratégica de marketing y su importancia, para el presente trabajo de investigación.

\section{Observación directa}

La técnica de la observación directa es un instrumento de recolección de información muy importante y consiste en el registro sistemático, válido y confiable de comportamientos o conducta manifiesta. Esta técnica se utilizó para establecer de forma clara y precisa la situación actual de las empresas y determinar donde se concentran los problemas de la planeación estratégica de marketing.

\section{Análisis estadístico}

\section{Método estadístico}

El método estadístico se lo utilizó para la recolección de la información necesaria, el mismo que facilitó el ordenar y clasificar los datos recolectados de las encuestas aplicadas a las empresas, a través de tablas y gráficos estadísticos representativos, el análisis y la interpretación estadística de los resultados para luego poder evidenciar la incidencia de sus respuestas y así se logró identificar los factores que guiaron la investigación.

\section{Resultados}

Análisis e interpretación de las encuestas aplicadas a las empresas privadas de la ciudad de Loja 
¿La empresa que Usted dirige aplica algún tipo de planeación estratégica para el desarrollo de sus actividades?

Tabla 1. Aplica algún tipo de planeación estratégica

\begin{tabular}{ccc}
\hline DESCRIPCIÓN & FRECUENCIA & PORCENTAJE \\
\hline Si & 178 & $68 \%$ \\
No & 82 & $32 \%$ \\
TOTAL & $\mathbf{2 6 0}$ & $\mathbf{1 0 0 \%}$
\end{tabular}

Fuente: Encuestas aplicadas a las empresas privadas de la ciudad de Loja Elaboración: La Autora

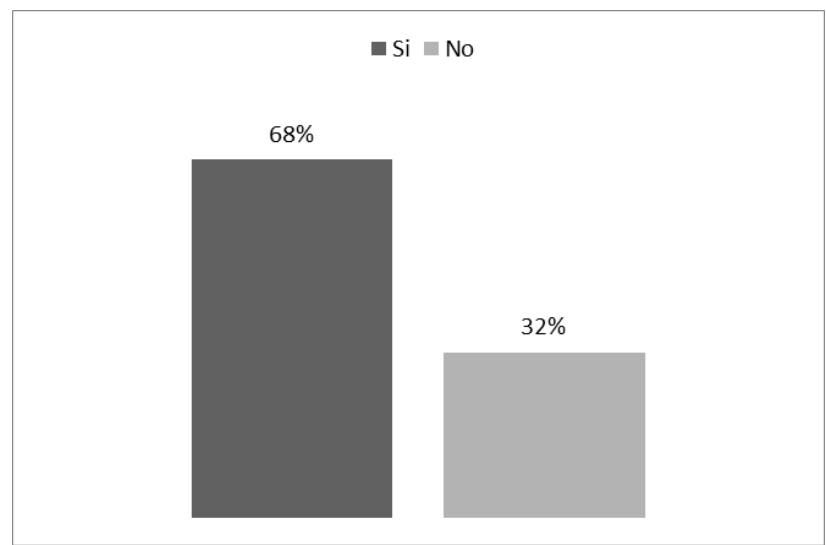

Figura 4. Aplica algún tipo de planeación estratégica Fuente: Tabla 1 Elaboración: La Autora

Análisis e interpretación: De las encuestas aplicadas a las empresas de la ciudad de Loja, el $68 \%$ de los encuestados manifestaron que si aplica algún tipo de planeación estratégica para el desarrollo de sus actividades; mientras que el 32\% mencionan su negatividad al respecto. Por lo tanto supieron manifestar que algunas empresas no la aplican, debido a que son negocios familiares y lo hacen empíricamente.

¿La planeación que Usted aplica se basa en objetivos y estrategias?

Tabla 2. Planeación en base a objetivos y estrategias

\begin{tabular}{lll}
\hline DESCRIPCIÓN & FRECUENCIA & PORCENTAJE \\
\hline Si & 161 & $62 \%$ \\
No & 99 & $38 \%$ \\
TOTAL & 260 & $100 \%$ \\
\hline
\end{tabular}

Fuente: Encuestas aplicadas a las empresas privadas de la ciudad de Loja Elaboración: La Autora 


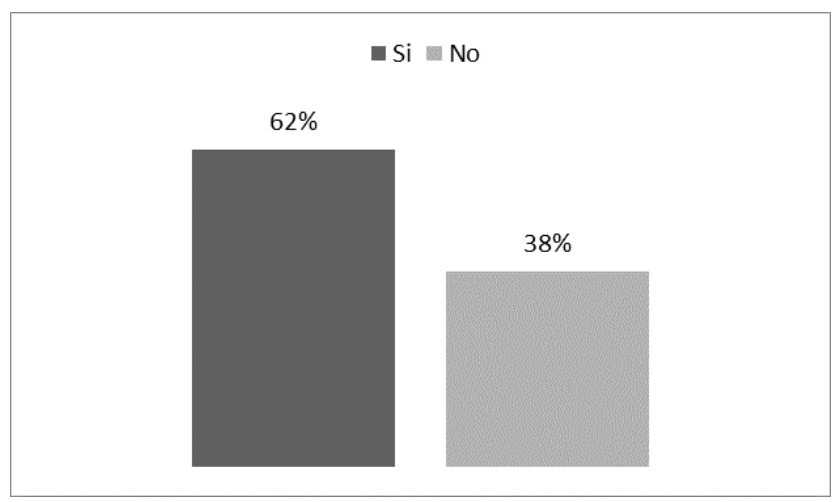

Figura 6. Planeación en base a objetivos y estrategias Fuente: Tabla 2.

Elaboración: La Autora

Análisis e interpretación: Del total de encuestados el 62\% afirmaron que la planeación que aplican se basa en objetivos y estrategias, en cambio el 38\% indicaron que no. Por lo que se evidenció que en un mayor porcentaje tienen conocimientos sobre los lineamientos a seguir en la planeación basada en objetivos y estrategias.

¿La empresa cuenta con misión y visión?

Tabla 3. Misión y visión

\begin{tabular}{ccc}
\hline DESCRIPCIÓN & FRECUENCIA & PORCENTAJE \\
\hline Si & 198 & $76 \%$ \\
No & 62 & $24 \%$ \\
TOTAL & 260 & $100 \%$
\end{tabular}

Fuente: Encuestas aplicadas a las empresas privadas de la ciudad de Loja Elaboración: La Autora

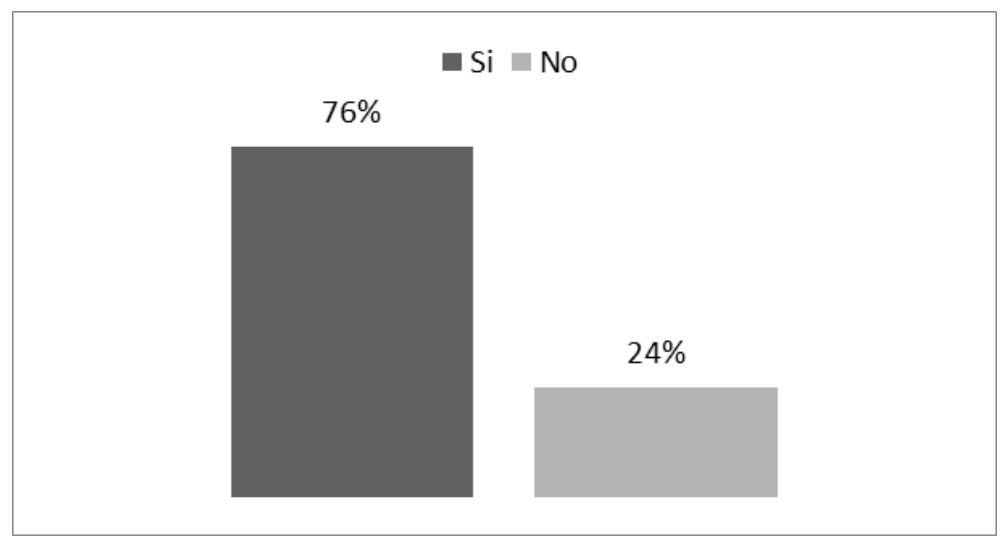

Figura 7. Misión y visión

Fuente: Tabla 3.

Elaboración: La Autora

Análisis e interpretación: Del total de la población encuestada correspondientes a las empresas privadas de la ciudad de Loja, el 76\% de los encuestados manifestaron que si cuentan con misión y visión las empresas, mientras que el 24\% expresaron que no cuentan. Por tanto, se analizó la importancia de contar con misión y visión en las empresas para mejorar su 
competitividad, estar a la vanguardia, mantenerse y crecer en este mundo tan globalizado y tecnológico, que exige un alto grado de preparación y destreza.

¿El tipo de planeación que aplica Usted en su empresa le ayuda como directivo a evaluar los riesgos que debe asumir en la toma de decisiones?

Tabla 4. La planeación le ayuda a evaluar riesgos en la toma de decisiones

\begin{tabular}{lll}
\hline DESCRIPCIÓN & FRECUENCIA & PORCENTAJE \\
\hline Si & 249 & $96 \%$ \\
No & 11 & $4 \%$ \\
TOTAL & 260 & $100 \%$
\end{tabular}

Fuente: Encuestas aplicadas a las empresas privadas de la ciudad de Loja

Elaboración: La Autora

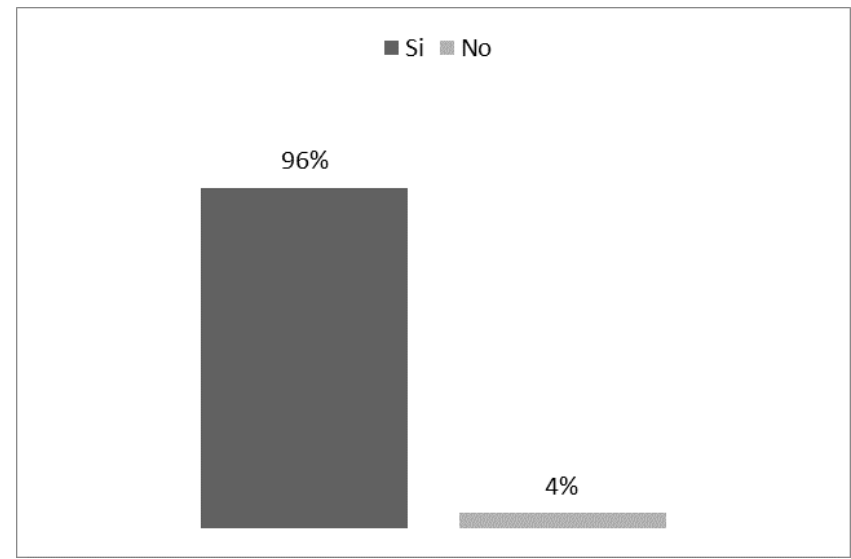

Figura 8. La planeación le ayuda a evaluar riesgos en la toma de decisiones Fuente: Tabla 4.

Elaboración: La Autora

Análisis e interpretación: En esta interrogante el total de los encuestados de las empresas privadas de la ciudad de Loja, el 96\% expresaron que el tipo de planeación que aplican en sus empresas les ayuda como directivos a evaluar los riesgos que deben asumir en la toma de decisiones, mientras que el $4 \%$ manifestaron que no. Se observó que la planificación estratégica de marketing es el centro de la actividad gerencial de una organización, es decir, sin un desarrollo correcto de esta función la empresa carece de orientación, definición y dirección.

¿Ha experimentado su empresa recientemente cambios políticos, económicos que hayan generado una toma de conciencia sobre los procesos de planificación estratégica?

Tabla 5. Factores externos y los procesos de planeación estratégica

\begin{tabular}{lll}
\hline DESCRIPCIÓN & FRECUENCIA & PORCENTAJE \\
\hline Si & 232 & $89 \%$ \\
No & 28 & $11 \%$ \\
TOTAL & 260 & $100 \%$
\end{tabular}

Fuente: Encuestas aplicadas a las empresas privadas de la ciudad de Loja Elaboración: La Autora 


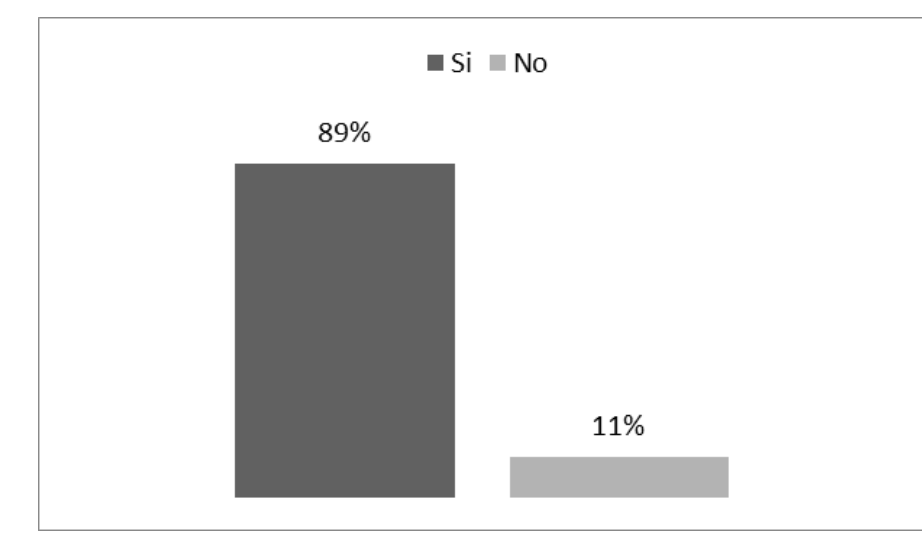

Figura 9. Factores externos y los procesos de planeación estratégica Fuente: Tabla 5.

Elaboración: La Autora

Análisis e interpretación: Del total de la población encuestada correspondientes a las empresas privadas de la ciudad de Loja, el 89\% de los encuestados manifestaron que si han experimentado en sus empresa recientemente cambios políticos y económicos que hayan generado una toma de conciencia sobre los procesos de planificación estratégica, mientras que el $11 \%$ expresaron que no. En este aspecto se evidenció que es necesario hacer conciencia sobre la planificación estratégica, para hacer frente a los aspectos externos de las empresas, es decir aprovechas las oportunidades sobre las amenazas.

$¿$ Cree Usted que es importante implementar planes estratégicos de marketing en las empresas, para su buen desenvolvimiento empresarial?

Tabla 6. Importancia de implementar planes estratégicos de marketing

DESCRIPCIÓN FRECUENCIA PORCENTAJE

\begin{tabular}{lll}
\hline Si & 260 & $100 \%$ \\
No & 00 & $0 \%$
\end{tabular}

$\begin{array}{lll}\text { TOTAL } & 260 & 100 \%\end{array}$

Fuente: Encuestas aplicadas a las empresas privadas de la ciudad de Loja Elaboración: La Autora

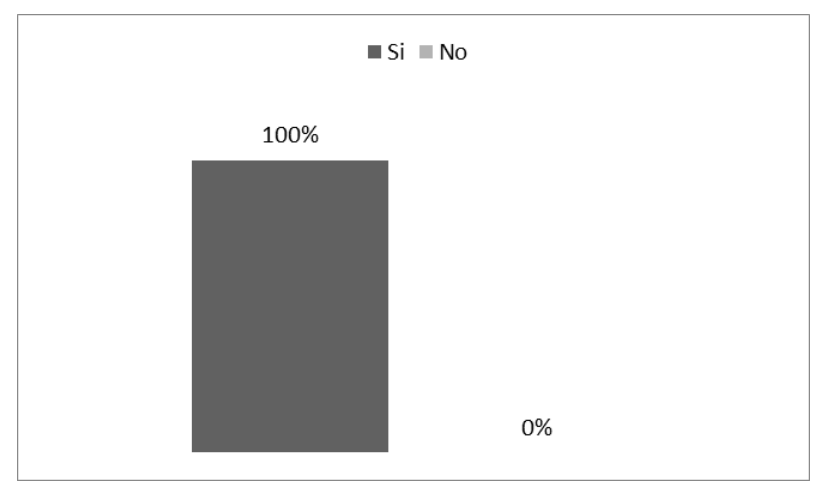

Figura 10. Importancia de implementar planes estratégicos de marketing Fuente: Tabla 6.

Elaboración: La Autora 
Análisis e interpretación: Del total de la población encuestada correspondientes a las empresas privadas de la ciudad de Loja, el 100\% de los encuestados afirmaron que si es importante implementar planes estratégicos de marketing en las empresas para su buen desenvolvimiento empresarial. Porque gracias a ello se puede planear un horizonte empresarial se puede lograr un objetivo con más calma ya que al planear se revelan los posibles errores y las ventajas con las que se cuenta al momento de realizar un proyecto.

\section{Discusión}

La propuesta de planeación estratégica de marketing se basa en los resultados de las encuestas aplicadas a las empresas de la ciudad de Loja, donde se determina que las empresas no aplican una adecuada planeación estratégica lo que ha dificultado el crecimiento y posicionamiento de las empresas de la ciudad de Loja.

Se realizó un estudio minucioso de los resultados de las encuestas aplicadas, el mismo que se pone a consideración delos directivos de las empresas para mejorar su desempeño laboral y a la vez contribuir con la sociedad mediante la presencia de nuevas fuentes de empleo.

En las empresas de la ciudad de Loja se evidencia la necesidad de aplicar los instrumentos y herramientas adecuadas de planificación para alcanzar propósitos, objetivos y metas, por lo que es conveniente plantear la propuesta en estudio. En donde, se busca obtener mayores beneficios a largo plazo y lograr mejores resultados de crecimiento en el mercado.

Es así, que es importante la implementación de modelos de planeación estratégica de marketing que ayuden a las empresas al cumplimiento y eficacia de los objetivos planteados, para ello es indispensable el compromiso total de los directivos de las empresas en la mejora continua de la toma de decisiones dentro de las organizaciones.

\section{Conclusiones}

En las empresas la planeación estratégica de marketing es una base fundamental porque gracias a ella se puede pronosticar alguna situación adversa y también porque sirve para implementar planes de acción en caso de contingencias, además de que al planear un horizonte empresarial se puede lograr un objetivo con más calma ya que al planear se revelan los posibles errores y las ventajas con las que se cuenta al momento de realizar un proyecto.

Evidentemente, la planeación estratégica conlleva a la promoción de nuevas formas de liderazgo, de trabajo en equipo y de colaboración entre los empleados; lo que demanda compromiso de cada uno de los integrantes de las empresas, mismos que deben tener en claro cuál es la misión, visión de futuro, y las metas que se proponen alcanzar.

La importancia de la planificación estratégica de marketing para las empresas consiste en el poder de anticipación, la iniciativa y la reacción oportuna de cambio, sustentando sus actos no en corazonadas sino en un plan, en el que se define los objetivos de la empresa y los procedimientos adecuados para alcanzarlos.

Finalmente se concluye que la planificación estratégica es importante ya que ayuda a fijar las prioridades y permite concentrarse en las fortalezas de las empresas, ayudando a tratar a los 
problemas de cambios en el entorno externo. Por tanto, depende de la planificación estratégica que se logren los objetivos empresariales con éxito.

\section{Bibliografía}

Arguin, G. (2000). La Planeación Estratégica En La Universidad. Segunda Edición.

Cutropía, F. C. (2003). Plan De Marketing Paso A Paso. España - Madrid: Editorial Esic.

Echeverri, C. L. (2008). Marketing Práctico. Colombia: 11va Ed., Mayol Ediciones S.A.

Ferrel, O., \& Hirt, G. (2004). Introducción A Los Negocios En Un Mundo Cambiante. México: McGraw Hill.

Fred, R. D. (1997). Conceptos De Administración Estratégica.

Koontz, H. D. (2010). Elementos De Administración. México: Sexta Edición, Editorial Macgraw Hill.

Kotler, P. (2000). Dirección De Marketing. Madrid: Edición Del Milenio, Prentice-Hall.

Kotler, P., \& Armstrong, G. (2004). Marketing. México: Cámara Dionisio Y Cruz Ignacio, Prentice Hall.

Munch, L. (2011). Planeación Estratégica: El Rumbo Hacia El Éxito. México: Trillas.

Porter, M. E. (2002). Ventaja Competitiva. Buenos Aires: S.L. (Grupo Patria Cultural) Alay Ediciones.

Renau, M. (2010). La Dirección Estratégica De La Empresa Un Enfoque Innovador Del Management. S.P.I.

Rojas, A. P. (2004). "Desarrollo Organizacional Y Gerencial": Un Enfoque Estratégico. Quito Ecuador: Segunda Edición.

Taylor, J. Y Machado, M. L. (2006) Higher Education Leadership and Management: From Conflict to Interdependence through Strategic Planning. Tertiary Education and Management, 12 (2), 137-160.

Thompson, A. A., Strickland, A. J., \& Gamble, E. (2012). Administración Estratégica. Madrid: Mac Graw Hill.

Uyarra, E. (2010) Conceptualizing the Regional Roles of Universities, Implications and Contradictions. European Planning Studies, 18 (8), 1227-1246.

World Bank (2002) Constructing Knowledge Societies: New Challenges for Tertiary Edu- Cation. A World Bank Report, Washington, Dc: The World Bank. 